\title{
Outcomes of the Perplexed Surgical Management of Retinal Detachment in Eyes with Coloboma
}

\author{
Simanta Khadka, Raghunandan Byanju, Sangita Pradhan \\ Department of Vitreo-Retina, Bharatpur Eye Hospital, Bharatpur, Nepal
}

Purpose: To determine the anatomical and visual outcomes of retinal detachment in eyes with chorioretinal coloboma managed by pars plana vitrectomy, endolaser photocoagulation and silicone oil (SO) tamponade.

Methods: Retrospective review of 29 eyes of 29 patients with retinal detachment associated with chorioretinal coloboma. All the cases were managed by vitrectomy procedures concluding with SO tamponade. Encircling band was placed based on pre-operative evaluation and/or surgeon's discretion. Endolaser photocoagulation was applied around the peripheral retina, all around the peripheral breaks and around the colobomatous area. The outcome measures were evaluated with regard to functional and anatomical success.

Results: The average age at the time of surgery was $21.76 \pm 9.58$ years (range, $10-50$ years). The mean follow-up duration was $12.28 \pm 4.8$ months (range, 6-24 months). Primary attached retina was obtained in 21 / 29 (72.4\%) eyes after single surgery. Re-detachment in 8 / 29 (27.6\%) eyes which required revision surgery was the most frequent postoperative complication followed by raised intraocular pressure in 4 / 29 (13.8\%) with SO in situ. Out of 29 eyes, 23 were followed up after the removal of SO. The mean duration of SO removal was $7.91 \pm 3.9$ months (range, 4-18 months). Implantation of encircling band, lens removal and cryotherapy provided no added advantage. At the final examination, improvement in vision was observed in 21 (72.4\%) eyes and the anatomical attachment of the retina was attained in 27 (93.1\%) eyes.

Conclusions: Complete pars plana vitrectomy, endolaser photocoagulation along with SO tamponade is effective for retinal detachment associated with chorioretinal coloboma. This technique improves the anatomical outcome and helps in regaining significant visual acuity.

Key Words: Coloboma, Retinal detachment, Silicone oils, Vitrectomy

Received: May 14, 2020 Final revision: October 5, 2020 Accepted: November 5, 2020

Corresponding Author: Simanta Khadka, MD. Department of VitreoRetina, Bharatpur Eye Hospital, Bharatpur-10, Chitwan, Nepal. Tel : 977056-521733, Fax : 977-056-523333,E-mail: behchitwan@beh.org.np

This study was presented at XXI Nepal Ophthalmic Society Conference on September, 2019 in Bhairahawa, Nepal and at International Council of Ophthalmology, World Ophthalmology Congress 2020 Virtual on 2629 June, 2020.
Chorioretinal (CR) coloboma is the congenital anomaly, attributed to the defect in the normal fusion of the embryonal fissure of the optic cup [1]. Genetic and environmental risk factors or triggers during an intrauterine period have been claimed to contribute to this entity [2]. Histopathologically, structural alterations in coloboma include staphylomatous sclera and deficiency of retinal pigment epithelium (RPE), Bruch's membrane, and choroid [3]. 
The optical coherence tomography evaluation of the margins of CR coloboma has revealed inner neurosensory retinal layers which continue as the intercalary membrane (ICM) in the area of coloboma, whereas the outer layers turn back, become disorganized, and fuse with the RPE [4]. The junction where this reversal occurs has been termed as "locus minoris resistentiae" [3,5]. Glial atrophy, schisis, hole formation in the ICM and disruption of the fluid flow barriers due to separation of the locus minoris resistentiae from the RPE is believed to be the mechanism of retinal detachment (RD) in CR coloboma. This process is further exacerbated by scleral ectasia, increasing vitreous traction at the margin, and vascular ischemia within the ICM [3].

The reported lifetime risk of RD is approximately $40 \%$ in eyes with CR coloboma $[1,6]$. The location of retinal breaks responsible for RD may be outside the area of the coloboma, inside the anomalous retinal tissue within the coloboma, or both [7]. The management of RD in these eyes differs considerably. Associated nystagmus may pose further difficulty in these type of eyes during preoperative evaluation. RD due to retinal break outside of the colobomatous area had been managed by conventional scleral buckling technique. However, detachment due to break in the hypoplastic retinal tissue within the colobomatous area may need different management approach [8]. The outcome of RD surgery in CR coloboma is not as gratifying as that in general population where anatomical success rate varies greatly. The factors that limit the success of surgery in these eyes include difficulty in identifying the break within the ICM, posterior location of the break and the absence of an underlying RPE which helps in creation of a CR adhesion around the break [5].

Advancement in pars plana vitrectomy (PPV) systems, wide-angle viewing systems, better illumination, and internal tamponading agents aid in better visualization of the retina, retinal breaks, and colobomatous areas, hence this approach is more favorable. In order to isolate the breaks and colobomatous area from the rest of the retina, retinopexy around the breaks and colobomatous margin and the use of internal tamponade to allow the treated area to develop CR adhesion is preferred [9]. Herein, we retrospectively reviewed a series of eyes seen at our practice with an aim to report our preferred method of surgery along with anatomical and functional outcome in eyes with $\mathrm{CR}$ coloboma undergoing RD surgery.

\section{Materials and Methods}

A retrospective chart review was performed for consecutive patients with rhegmatogenous RD in eyes with $\mathrm{CR}$ coloboma who presented to our tertiary eye care center from January 2014 to July 2019. The study was approved by the institutional review committee of Bharatpur Eye Hospital (174/076/77). Written informed consent was waived due to the retrospective nature of the study. The study was performed in accordance with the tenets of the Declaration of Helsinki.

The medical records were reviewed to collect the following data: age, gender, laterality, ocular status, history of trauma, duration of symptom, slit-lamp biomicroscopy and indirect ophthalmoscopy examination findings, associated ocular or systemic abnormalities, clinical characteristics of RD and coloboma, visual acuity (VA), intra ocular pressure (IOP), surgical procedures, postoperative complications if any, revision of surgery if required and follow-up duration. The CR coloboma was categorized as per Ida Mann classification, which is stated as follows [10]; type 1: coloboma extending above the anatomic disc, type 2: coloboma extending up to the superior border of disc, type 3 : coloboma extending below the lower border of disc, type 4 : coloboma involving the disc only, type 5: coloboma present below the disc with normal retina above and below the coloboma, type 6: pigmentation present in the periphery, and type 7: coloboma involving only the periphery.

All the consecutive patients with RD in eyes associated with coloboma with retinal break in either colobomatous or extra-colobomatous region were included. Patients with retinal problems other than RD were excluded from the study. All eyes underwent standard three port 20 - or 23-gauge PPV with silicone oil (SO) tamponade at the conclusion of surgery. Hypotony was defined as persistent IOP of $5 \mathrm{mmHg}$ or less at four weeks following surgery. An IOP $25 \mathrm{mmHg}$ or higher was considered elevated. Anatomical success was defined as attachment of sensory retina with RPE with no residual subretinal fluid. Similarly, functional success was defined as any or slight improvement in VA at the final follow-up compared to vision at presentation. The collected data were analyzed in IBM SPSS Statistics ver. 20 (IBM Corp., Armonk, NY, USA). Qualitative variables were reported using frequency and percentage. Quantitative data were defined using mean \pm standard deviation. Fisher's exact test was applied for cate- 

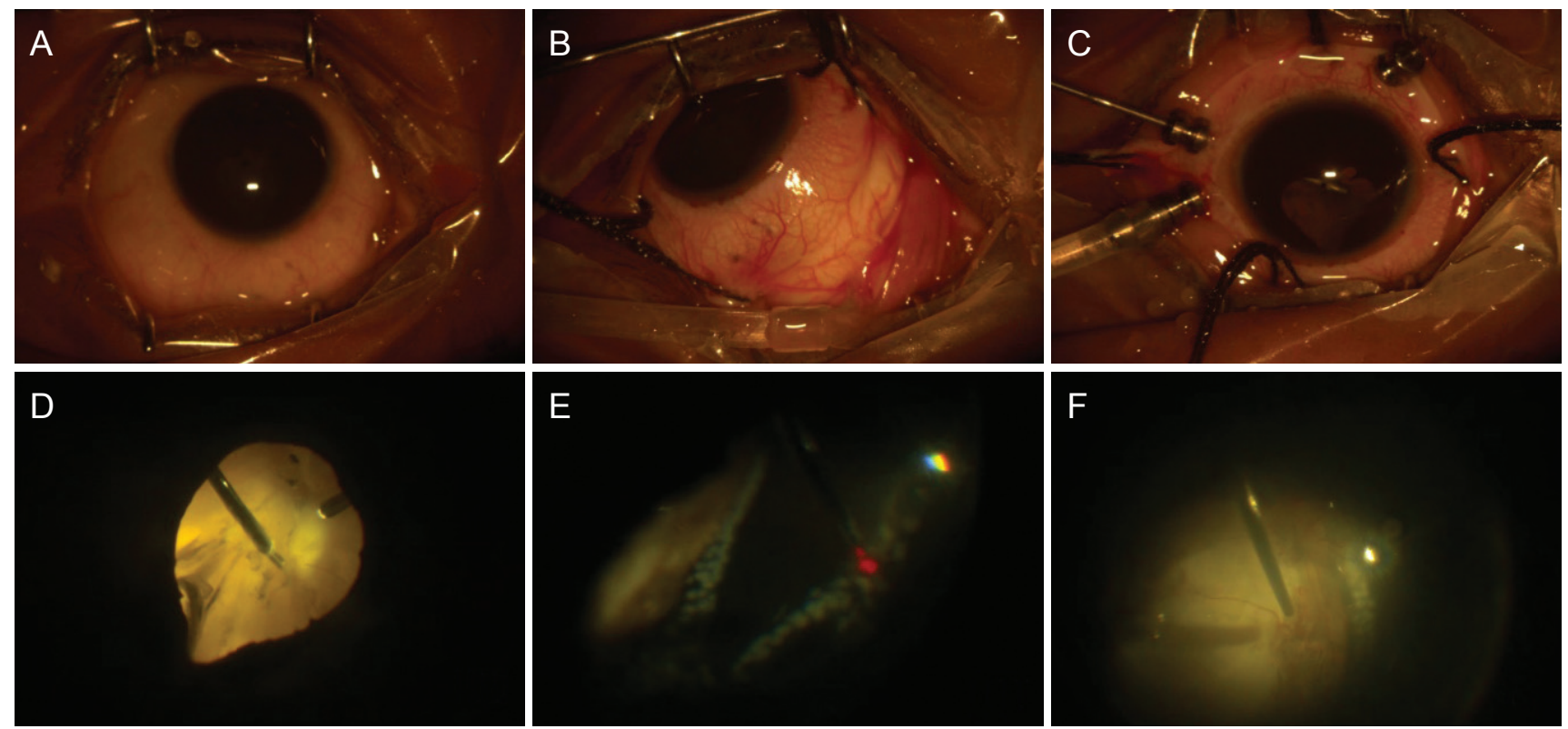

F

Fig. 1. Details of intraoperative procedures. (A) On table appearance of the eye and showing typical iris coloboma. (B) Encircling band buckle implantation. (C) Pars plana lensectomy. (D) Membrane peeling. (E) Endolaser application along the margin of coloboma as well as peripheral retina. (F) Fluid air exchange.
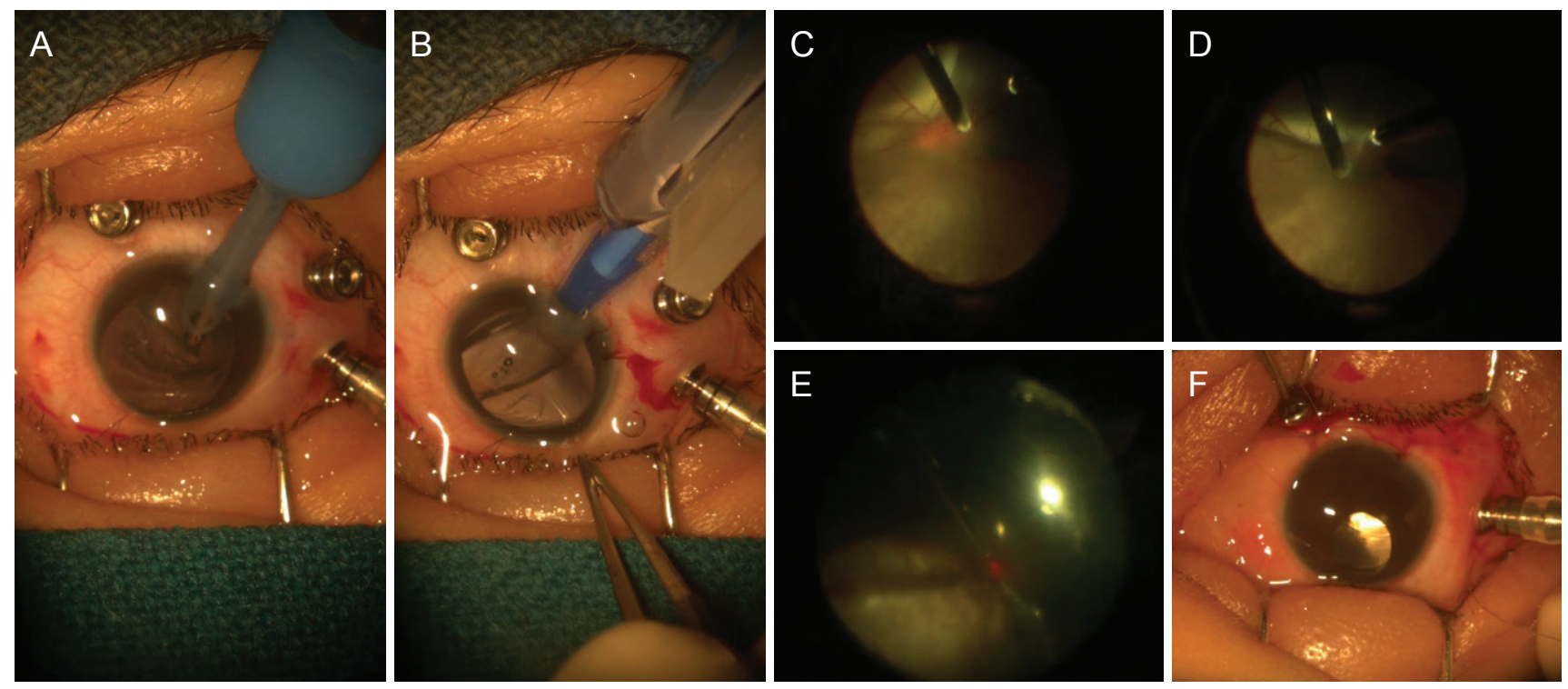

Fig. 2. Intraoperative details of another patient. (A) Lens aspiration with automated irrigation aspiration cannula. (B) Implantation of foldable posterior chamber intraocular lens. (C) Type 5 chorioretinal coloboma with visible detached retina. (D) Detachment of posterior hyaloid with active suction by vitreous cutter. (E) Endophotocoagulation laser application. (F) Silicone oil instillation at conclusion.

gorical variables. Wilcoxon signed rank test was used for the analysis of categorical data for comparing preoperative and postoperative VA. A $p$-value was calculated at $95 \%$ confidence interval and $p$-value $<0.05$ was considered statistically significant. VA documented in Snellen's chart values were converted to logarithm of the minimum angle of resolution ( $\log$ MAR) equivalent for analysis. A modified scale was used for "counting fingers," "hand movements," "perception of light," and "no perception of light" and assigned non-numerical $\log$ MAR scores of 2.0, 2.7, 3.0, 
and 4.0 respectively [11].

All surgical procedures were performed under peribulbar anesthesia or general anesthesia. The patients underwent a standard 20- or 23-gauge PPV procedure using a wide-angle viewing system with or without lensectomy. A lensectomy was performed if the lens was found to obscure the view of fundus due to cataract or impede adequate management of peripheral vitreous. Posterior chamber intraocular lens was implanted whenever possible. After removal of the anterior and core vitreous, triamcinolone acetonide aqueous suspension was injected into the mid-vitreous cavity for visualization of the residual vitreous and posterior hyaloid membrane. Posterior vitreous detachment induction was executed by suction using the vitreous cutter hand piece or a silicone-tipped cannula over the edges of the optic nerve head. The cortical vitreous was removed extensively relieving the vitreous traction around the retinal breaks, and the vitreous base was shaved after meticulous scleral indentation. Depending on the intraoperative situation, additional procedures; cryopexy and/or the use of an encircling band (No. 240) were performed during PPV. The retina was flattened by instillation of perfluorocarbon liquid or by fluid-air exchange followed by internal drainage of subretinal fluid through either an already existing break or a drainage retinotomy. If the peripheral vitreoretinal tractions in cases with advanced proliferative vitreo-retinopathy did not allow retinal reattachment, then relaxing retinotomy/retinectomy was performed. All eyes underwent laser endophotocoagulation performed in three to four rows all around the peripheral retina along the vitreous base, around the peripheral breaks and all around the colobomatous area (Fig. 1A-1F). The surgery was completed by air-oil exchange and instillation of 1,000 centistokes SO (Fig. 2A-2F). An inferior peripheral iridectomy was performed with vitreous cutter to prevent pupillary block in all aphakic cases. Sclerotomy ports and the conjunctiva were closed with 8-0 vicryl suture. During the postoperative period, patients were advised to keep head positioning defined by the break location for at least seven days. The SO was removed using a 3-port, 20-gauge technique through either limbal or pars plana approach under general or peribulbar anesthesia.

\section{Results}

A total of 29 eyes of 29 consecutive patients were included in this study. The average age at surgery was $21.8 \pm$ 9.6 years (range, $10-50$ years). No history of consanguinity was present in the patients of our series. Previous history of barrage laser treatment along the margin of coloboma

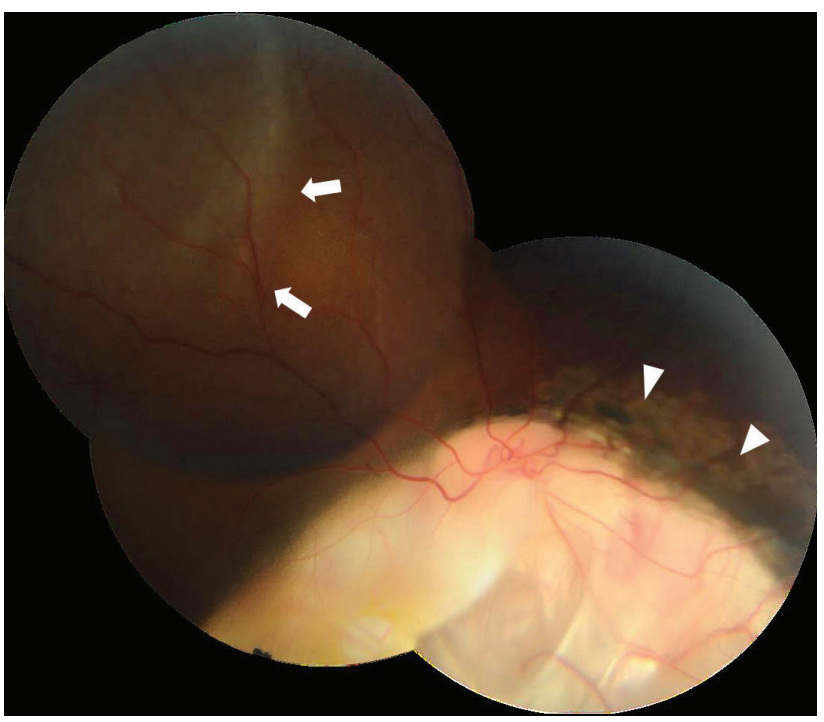

Fig. 3. Type 2 chorioretinal coloboma of right eye as per Ida Mann classification. The detached retina is shown with white arrows and previous scars of barrage laser treatment along the colobomatous margin is shown by arrowhead. The break was identified within the intercalary membrane intraoperatively.

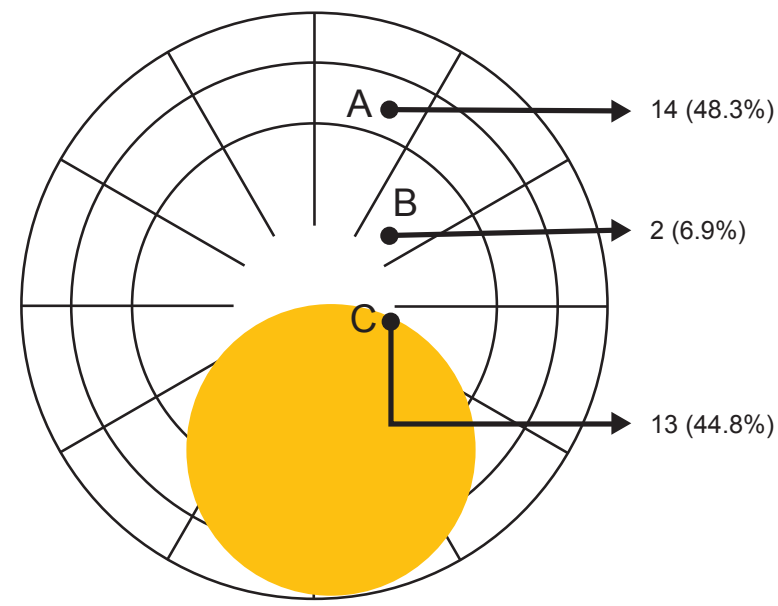

Fig. 4. Determined location of retinal breaks. A, retinal periphery; $\mathrm{B}$, posterior to the equator and within the normal retina; $\mathrm{C}$, break in the colobomatous region or at the edge of the coloboma (intercalary membrane). 
Table 1. Baseline clinical features

\begin{tabular}{|c|c|c|}
\hline Parameter & Frequency & Percentage \\
\hline \multicolumn{3}{|l|}{ Sex } \\
\hline Male & 22 & 76 \\
\hline Female & 7 & 24 \\
\hline \multicolumn{3}{|c|}{ Duration of symptoms at presentation } \\
\hline$<1$ mon & 3 & 10.3 \\
\hline $1-3$ mon & 1 & 3.4 \\
\hline $3-6$ mon & 5 & 17.2 \\
\hline 6 mon-1 yr & 10 & 34.5 \\
\hline$>1 \mathrm{yr}$ & 10 & 34.5 \\
\hline \multicolumn{3}{|l|}{ Laterality } \\
\hline Right eye & 15 & 51.7 \\
\hline Left eye & 14 & 48.3 \\
\hline One eyed status & 10 & 34.5 \\
\hline \multicolumn{3}{|c|}{ Associated ocular abnormalities } \\
\hline Iris coloboma & 27 & 93 \\
\hline Microcornea & 5 & 17.4 \\
\hline Microphthalmos & 3 & 10.4 \\
\hline Fellow eye phthisis & 3 & 10.4 \\
\hline \multicolumn{3}{|c|}{ Classification of coloboma } \\
\hline Type 1 & 5 & 17.2 \\
\hline Type 2 & 9 & 31 \\
\hline Type 3 & 11 & 38 \\
\hline Type 4 & - & - \\
\hline Type 5 & 4 & 13.8 \\
\hline Type 6 & - & - \\
\hline Type 7 & - & - \\
\hline
\end{tabular}

was present in 4 / 29 (13.8\%) eyes (Fig. 3). The baseline clinical features are summarized in Table 1.

The retinal breaks found in the region of coloboma were 13 / 29 (44.8\%) and the incident retinal breaks is depicted in Fig. 4. Out of these breaks in colobomatous region, preoperatively break was determined in only 5 / 13 (38.5\%) eyes and in remaining $8 / 13$ (61.5\%) eyes, the break was identified intraoperatively.

Summary of intraoperative additional surgical procedure is shown in Table 2. These additional procedures provided no additional advantage to the final anatomical outcome $(p>0.05)$. Following lensectomy, posterior chamber intraocular lens implantation was possible in only $3 / 29$ $(10.3 \%)$ eyes.

The patients were followed up for at least six months duration postsurgery with an average of $12.28 \pm 4.8$ months (range, 6-24 months). The cases that underwent SO removal 23 / 29 (79.3\%) was performed at an average of 7.9 \pm 3.9 months (range, 4-18 months) (Table 3). Six patients didn't follow-up for further evaluation but the retina remained attached in those patients at the end of last visit.

Table 3. Indications for silicone oil removal $(\mathrm{n}=23)$

\begin{tabular}{lcc}
\hline Indication & Frequency & Percentage \\
\hline Effective tamponade & 15 & 65.2 \\
Raised intraocular pressure & 4 & 17.4 \\
Silicone oil emulsification & 4 & 17.4 \\
\hline
\end{tabular}

Table 2. Predictors of final anatomical outcome following additional surgical procedures

\begin{tabular}{|c|c|c|c|c|c|c|}
\hline & & Attached $(n=27)$ & Detached $(n=2)$ & Total $(n=29)$ & $p$-value ${ }^{*}$ & Risk estimate \\
\hline \multirow[t]{2}{*}{ Lensectomy } & Done & $21(91.3)$ & $2(8.7)$ & $23(100)$ & 0.99 & $0.91^{\dagger}$ \\
\hline & Not done & $6(100)$ & $0(0)$ & $6(100)$ & & \\
\hline \multirow[t]{2}{*}{ Encircling band (No. 240) } & Implanted & $13(92.9)$ & $1(7.1)$ & $14(100)$ & 0.99 & $0.93^{*}$ \\
\hline & Not implanted & $14(93.3)$ & $1(6.7)$ & $15(100)$ & & \\
\hline \multirow[t]{2}{*}{ Cryotherapy } & Applied & $10(90.9)$ & $1(9.1)$ & $11(100)$ & 0.99 & $0.59^{\ddagger}$ \\
\hline & Not applied & $17(94.4)$ & $1(5.6)$ & $18(100)$ & & \\
\hline \multirow[t]{2}{*}{ Perfluorocarbon liquid } & Used & $11(100)$ & $0(0)$ & $11(100)$ & 0.51 & $1.1^{\dagger}$ \\
\hline & Not used & $16(88.9)$ & $2(11.1)$ & $18(100)$ & & \\
\hline \multirow[t]{2}{*}{ Silicone oil removal } & Performed & $21(91.3)$ & $2(8.7)$ & $23(100)$ & 0.99 & $0.91^{\dagger}$ \\
\hline & Not performed & $6(100)$ & $0(0)$ & $6(100)$ & & \\
\hline
\end{tabular}

Values are presented as number (\%).

"Fisher's exact test; ${ }^{\dagger}$ Relative risk, percentage mentioned in parentheses; ${ }^{\ddagger}$ Odds ratio. 
Table 4. Postoperative complications $(n=13)$

\begin{tabular}{lccl}
\hline Indication & Frequency & Percentage & Subsequent management \\
\hline Re-detachment & 8 & 27.6 & Revision surgery \\
Raised intraocular pressure & 4 & 13.8 & SO removal \\
Hypotony after SO removal & 1 & 3.4 & Posterior subtenon triamcinolone 20 mg \\
\hline
\end{tabular}

$\mathrm{SO}=$ silicone oil

Table 5. Summary of surgical outcome at the final follow-up $(\mathrm{n}=29)$

\begin{tabular}{lcc}
\hline Parameter & Frequency & Percentage \\
\hline Anatomical outcome & & \\
$\quad$ Retinal re-attachment & 27 & 93.1 \\
$\quad$ Detached retina & 2 & 6.9 \\
Functional outcome & & \\
$\quad$ Improvement in VA & 21 & 72.4 \\
$\quad$ Static or deterioration of VA & 8 & 27.6 \\
\hline
\end{tabular}

$\mathrm{VA}=$ visual acuity.

${ }^{*}$ Wilcoxon signed rank test.

Primary anatomical success was attained in $21 / 29$ (72.4\%) eyes and revision of the surgery was required in 8 / $29(27.6 \%)$ patients with mean duration of $7.25 \pm 2.5$ months (range, 4-12 months) for re-detachment following primary PPV. Out of these patients, six patients were implanted with encircling band accounting for $42.8 \%$ of revision rate among 14 patients with encircling band. During revision, retinectomy was required in two of eight eyes $25 \%$ for advanced proliferative vitreo-retinopathy. Superior retinectomy was performed where one eye required two clock hours retinectomy from 12 to 2 o'clock, similarly another eye required retinectomy from 10 to 2 o'clock hours. Complete retinal re-attachment had been achieved in 27 (93.1\%) patients and two (6.9\%) eyes remained detached at the final visit. Similarly, the vision was improved in $21 / 29$ (72.4\%) patients. Five out of 29 (17.2\%) patients had similar antecedent vision and deteriorated vision was recorded in $3 / 29$ (10.4\%) patients at the end of final visit. Out of these three, decreased vision was due to raised IOP and secondary glaucoma in two patients and one eye progressed to phthisis bulbi. The documented vision in these cases were no perception of light. Re-detachment in 8 / 29 (27.6\%) eyes was the most frequent postoperative complication fol- lowed by raised IOP in 4 / 29 (13.8\%) eyes with SO in situ. Hypotony (3.4\%) was encountered in a single eye following SO removal, but the IOP was maintained within normal range at the last follow-up. The postoperative complications is shown in Table 4. The mean postoperative VA improved to $1.89 \pm 1.03 \log$ MAR ( $1 / 60$ in Snellen equivalent) compared to preoperative VA of $2.72 \pm 0.4 \log$ MAR (hand movement in Snellen equivalent) which was statiscally significant $(p<0.0001)$. The summary of anatomical and functional success is summarized in Table 5 .

\section{Discussion}

$\mathrm{RD}$ in eyes with CR coloboma is a rare clinical entity [12]. The RD in this scenario pose a challenge to repair [6]. In our study, the overall anatomic success with a mean follow-up duration of $12.28 \pm 4.8$ months was 27 of the 29 eyes (93.1\%). The rate of recurrent RD after primary PPV was 8 / 29 eyes (27.6\%). Functional achievement in terms of vision improvement was achieved in 21 out of 29 eyes (72.4\%). Though not improved, the VA was preserved to that of previous state in 5 / 29 (17.2\%) eyes and the vision deteriorated to denial of perception of light in $3 / 29$ (10.4\%) eyes.

The anatomic success of our study is comparable to another series of ten eyes where the reported anatomic attachment was $90 \%$, but it had a relative longer follow-up duration of $28.8 \pm 28.4$ months (range, 9-101 months) [9]. Similarly, anatomical re-attachment of $87.4 \%$ with an average follow-up of $41 \pm 36.5$ months was present in 119 eyes [13]. Other reports in the literature mention success rates that ranged from $35-100 \%$ at the end of follow up visit $[14,15]$. VA in terms of functional achievement in our study was $72.4 \%$, which is proportionate to another report of $78.4 \%$ with inclusion of 42 eyes of 40 patients [15]. The postoperative mean VA reported was $1.4 \pm 1.6 \log$ MAR at 
the last follow-up [13], whereas our study showed a mean VA of $1.89 \pm 1.03 \log$ MAR. Though acceptable anatomical attachment was attained, the result of involvement of vital structures (retina, optic disc, and macula) in the eye as well as pre-existing amblyopia might influence the final visual outcome [16].

Although coloboma can occur in isolation, they are commonly associated with other congenital ocular and systemic anomalies [17]. Our cases do not reveal any systemic abnormalities upon review. However, being a component of typical coloboma, our series also had iris coloboma as a major-associated ocular anomalies [18]. The retinal breaks in eyes with detachment identified outside the margin of the coloboma may be managed with conventional scleral buckling techniques, however the anatomical success rates reported ranged from $35 \%$ to $57 \%$ which is not so encouraging $[1,19]$. We identified the retinal break in 16 eyes; 14 in the far periphery and two in region posterior to the equator and within the normal retina which are away from the colobomatous margin, we preferred PPV with long term SO tamponade in these cases as well. Most reports on scleral buckle surgery in colobomatous eyes date back to earlier decades and it is believed that colobomatous eyes are not suitable for conventional buckle surgery [20]. RD was attributed to the coloboma when the extension of the detachment was within the border of the coloboma [5], which was present in 13 eyes in our study. Similarly, we didn't find any significant association that can influence the final anatomical outcome with regard to lens extraction, implantation of band buckle, cryotherapy or use of perfluorocarbon liquid $(p>0.05)$. In accordance with our result, a study also advocated no additional benefit of encircling band in RD associated with CR coloboma [5]. As the total number of patients in our series is small, statistical significant difference was not obtained in any of the additional procedure performed. However, intraoperative use of perfluorocarbon liquid is assocaited with 1.1 fold increase in risk of RD.

Off all the patients, revision surgery was required in $8 /$ 29 (27.6\%) patients. Among them six patients were implanted with encircling band based on the location of the break which was found to be away from the margin of coloboma. The fact that the eyes that developed recurrent RD even when they were filled with SO and had a predetermined break within the normal retina indicates the complexity of detachment in these cases [13]. This reiterate the possibility of an abnormal vitreoretinal interface in colobomatous eyes which may not be necessarily limited to the area of the coloboma and its margins [21]. An importance to consider a break within the ICM and at the edge of the coloboma (locus minoris resistentiae) is suggested in any $\mathrm{RD}$ in a colobomatous eye with only an identifiable break in the normal retinal periphery. There is also likelihood of iatrogenic breaks at the edge of coloboma due to tractional forces on the retina during the induction of posterior vitreous detachment [5]. In these cases, meticulous identification and treatment of all the peripheral break and sealing the edge of the coloboma from the remaining retina needs to be performed even if no break was identified in the ICM. We prefer endolaser photocoagulation application in three to four rows, around the identified peripheral breaks and along the margin of CR coloboma as well as along the vitreous base to cover the unidentified breaks in the retinal periphery. Regardless of the advancement in visualization system, identifying a break within the ICM intraoperatively is difficult [13]. Hence a high index of suspicion not to miss the break and careful examination of possible area is mandated.

Alternative was explored with the use of cyanoacrylate glue for sealing the break in ICM [22]. Pneumatic retinopexy alone followed by photocoagulation laser around the margin of coloboma was successful to re- attach the retina in few instances $[23,24]$. However, larger series and data from a longer follow-up for these alternatives is yet to be validated. A long-term tamponade in the form of long-acting gas or SO is considered the standard of care for RD in eyes with coloboma [5].

SO removal in our study was performed in $23 / 29$ (79.3\%) eyes at an average of $7.9 \pm 3.9$ months (range, $4-18$ months). A study revealed SO removal in 55 / 119 (60\%) eyes after a mean duration of $15.6 \pm 19.8$ months. The documented SO related complications in literature were; SO migration into the anterior chamber, medically uncontrolled IOP mandating surgical intervention and cataract progression necessitating phacoemulsification [13]. We encountered SO emulsification in 4 / 23 (17.4\%) and persistent IOP rise in 4 / 23 (17.4\%). Total loss of vision (no perception of light) resulted due to secondary glaucoma in two eyes. Other corneal complications [8] and previously mentioned SO related complication was not reported in our study. Interestingly, SO induced cataract progression was not noted as lens extraction was performed in majori- 
ty of (79.3\%) eyes in our study during PPV.

The limitations of our study are several which includes its retrospective nature, small sample size, short follow-up duration and single center-based study. Sequestration bias might have influenced the higher rate of complex detachment to be managed in our institution which serves as a tertiary referral center for vitreoretinal diseases in this region. Studies with larger samples and longer follow up period is recommended before affirming a definitive statement.

In conclusion, our experience from PPV with SO tamponade and endolaser photocoagulation around the edge of the coloboma, all around the peripheral retina in addition to the primary identified breaks provided the best outcome for RD in eyes with $\mathrm{CR}$ coloboma. The anatomical results are excellent with this technique. Additionally, this surgical modality also helped to enhance the mobility of the patient, and significant improvement in vision was achieved which ultimately improves the quality of life.

\section{Conflict of Interest}

No potential conflict of interest relevant to this article was reported.

\section{References}

1. Wang K, Hilton GF. Retinal detachment associated with coloboma of the choroid. Trans Am Ophthalmol Soc 1985;83:4962.

2. Gregory-Evans CY, Williams MJ, Halford S, Gregory-Evans K. Ocular coloboma: a reassessment in the age of molecular neuroscience. J Med Genet 2004;41:881-91.

3. Schubert HD. Structural organization of choroidal colobomas of young and adult patients and mechanism of retinal detachment. Trans Am Ophthalmol Soc 2005;103:457-72.

4. Gopal L, Khan B, Jain S, Prakash VS. A clinical and optical coherence tomography study of the margins of choroidal colobomas. Ophthalmology 2007;114:571-80.

5. Gopal L, Badrinath SS, Sharma T, et al. Surgical management of retinal detachments related to coloboma of the choroid. Ophthalmology 1998;105:804-9.

6. Jesberg DO, Schepens CL. Retinal detachment associated with coloboma of the choroid. Arch Ophthalmol 1961;65:163-
73.

7. Michel R, Wilkinson C, Rice T. Retinal detachment. St Louis: CV Mosby; 1990. p. 117.

8. Gopal L, Kini MM, Badrinath SS, Sharma T. Management of retinal detachment with choroidal coloboma. Ophthalmology 1991;98:1622-7.

9. Hocaoglu M, Karacorlu M, Ersoz MG, et al. Outcomes of vitrectomy with silicone oil tamponade for management of retinal detachment in eyes with chorioretinal coloboma. Retina 2019;39:736-42.

10. Mann I. Developmental abnormalities of the eye. Philadelphia: Lippincott; 1957. p. 81-103.

11. Kitano M, Taneda M, Nakao Y. Postoperative improvement in visual function in patients with tuberculum sellae meningiomas: results of the extended transsphenoidal and transcranial approaches. J Neurosurg 2007;107:337-46.

12. Gopal L, Badrinath SS, Kumar KS, et al. Optic disc in fundus coloboma. Ophthalmology 1996;103:2120-6.

13. Abouammoh MA, Alsulaiman SM, Gupta VS, et al. Surgical outcomes and complications of rhegmatogenous retinal detachment in eyes with chorioretinal coloboma: the results of the KKESH International Collaborative Retina Study Group. Retina 2017;37:1942-7.

14. Daufenbach DR, Ruttum MS, Pulido JS, Keech RV. Chorioretinal colobomas in a pediatric population. Ophthalmology 1998;105:1455-8.

15. Pal N, Azad RV, Sharma YR. Long-term anatomical and visual outcome of vitreous surgery for retinal detachment with choroidal coloboma. Indian J Ophthalmol 2006;54:858.

16. Zafar SA, Qureshi NA, Pathan AH, et al. Surgical and visual outcomes of retinal detachment surgery in eyes with chorio-retinal coloboma. Pak Armed Forces Med J 2016;66:795-99.

17. Bermejo E, Martinez-Frias ML. Congenital eye malformations: clinical-epidemiological analysis of 1,124,654 consecutive births in Spain. Am J Med Genet 1998;75:497-504.

18. Onwochei BC, Simon JW, Bateman JB, et al. Ocular colobomata. Surv Ophthalmol 2000;45:175-94.

19. Ramezani A, Dehghan MH, Rostami A, et al. Outcomes of retinal detachment surgery in eyes with chorioretinal coloboma. J Ophthalmic Vis Res 2010;5:240-5.

20. Teoh SC, Mayer EJ, Haynes RJ, et al. Vitreoretinal surgery for retinal detachment in retinochoroidal colobomata. Eur J Ophthalmol 2008;18:304-8.

21. Hussain RM, Abbey AM, Shah AR, et al. Chorioretinal coloboma complications: retinal detachment and choroidal 
neovascular membrane. J Ophthalmic Vis Res 2017;12:3-10.

22. Hotta K, Hirakata A, Hida T. The management of retinal detachments associated with choroidal colobomas by vitrectomy with cyanoacrylate retinopexy. Jpn J Ophthalmol 1998;42:323-6.

23. Giansanti F, Giuntoli M, Mazzini C, et al. Pneumatic retinopexy for retinal detachment associated with choroidal coloboma. Eur J Ophthalmol 2012;22:680-2.

24. Rishi E, Rishi P, Govindarajan MV. Pneumatic retinopexy for the treatment of shallow retinal detachment caused by a retinal break in the intercalary membrane of macula sparing retinochoroidal coloboma. Retin Cases Brief Rep 2016;10:187-90. 